\title{
Real-space imaging of phase transitions in bridged artificial kagome spin ice
}

\section{Kevin Hofhuis ( $\square$ khofhuis@gmail.com )}

ETH Zurich - Paul Scherrer Institute https://orcid.org/0000-0002-1642-4786

\section{Sandra Skjærvø}

ETH Zurich - Paul Scherrer Institute

\section{Sergii Parchenko}

Paul Scherrer Institute

Hanu Arava

ETH Zurich - Paul Scherrer Institute

\section{Zhaochu Luo}

ETH Zurich/ Paul Scherrer Institute

\section{Armin Kleibert}

Paul Scherrer Institut https://orcid.org/0000-0003-3630-9360

\section{Peter Derlet}

Paul Scherrer Institute

\section{Laura Heyderman}

ETH Zurich - Paul Scherrer Institute https://orcid.org/0000-0003-3843-6611

\section{Article}

Keywords: magnetic phase transitions, kagome spin ice, artificial spin systems

Posted Date: September 28th, 2021

DOI: https://doi.org/10.21203/rs.3.rs-871750/v1

License: (c) (1) This work is licensed under a Creative Commons Attribution 4.0 International License. Read Full License

Version of Record: A version of this preprint was published at Nature Physics on April 4th, 2022. See the published version at https://doi.org/10.1038/s41567-022-01564-5. 


\title{
Real-space imaging of phase transitions in bridged artificial kagome spin ice
}

\author{
Kevin Hofhuis, ${ }^{1,2 *}$ Sandra Helen Skjærv $\varnothing,{ }^{1,2}$ Sergii Parchenko, ${ }^{1,2,3}$ Hanu Arava, ${ }^{1,2}$ Zhaochu Luo, ${ }^{1,2}$ \\ Armin Kleibert, ${ }^{3}$ Peter Michael Derlet, ${ }^{1,4}$ and Laura Jane Heyderman ${ }^{1,2^{*}}$ \\ ${ }^{1}$ Laboratory for Mesoscopic Systems, Department of Materials, ETH Zurich, 8093 Zurich, Switzerland \\ ${ }^{2}$ Laboratory for Multiscale Materials Experiments, Paul Scherrer Institute, 5232 Villigen PSI, Switzerland \\ ${ }^{3}$ Swiss Light Source, Paul Scherrer Institute, 5232 Villigen PSI, Switzerland \\ ${ }^{4}$ Condensed Matter Theory Group, Paul Scherrer Institute, 5232 Villigen PSI, Switzerland \\ e-mail: kevin.hofhuis@psi.ch, laura.heyderman@psi.ch
}

In frustrated spin systems, magnetic phase transitions underpin the formation of exotic, frustrationdriven magnetic phases. Of great importance is the ability to manipulate these transitions to access specific phases, which in turn provides a means to discover and control novel phenomena. Artificial spin systems, incorporating lithographically-fabricated arrays of dipolar-coupled nanomagnets that allow real-space observation of the magnetic configurations, provide such an opportunity. In particular, the kagome spin ice is predicted to have two phase transitions, one of which is to a low temperature phase whose long-range ground state order has not been observed experimentally. To achieve this order, we change the global symmetry of the artificial kagome system, by selectively tuning the near-field nanomagnet interactions through nanoscale bridges at the vertices. By precisely tuning the interactions, we can quantify the influence of frustration on such a transition, and find that the driving force for spin and charge ordering depends on the degeneracy strength at the vertex. For the first time, we are able to observe the evolution of the magnetic configurations associated with a phase transition in real space and time.

The ability to lithographically pattern dipolar-coupled monodomain nanomagnets into almost any lattice geometry has motivated the study and development of a wide variety of model spin systems that are in general referred to as artificial spin ice ${ }^{1-4}$. Although the magnetic configurations in these systems do not always mimic the ordering of bonds in water ice, they display an abundance of fascinating phenomena, including freely moving emergent magnetic monopoles ${ }^{5,6}$, topologically induced frustration in the Shakti lattice ${ }^{7,8}$, and phase coexistence ${ }^{9}$. The high degeneracy of magnetic states found in such frustrated systems can also be exploited in reservoir computing schemes ${ }^{10-12}$, although reaching the long-range ordered states with thermally-driven nanomagnets for such applications remains challenging. This is because the superparamagnetic blocking temperature is often above the critical temperature, particularly in highly frustrated systems such as the artificial kagome spin ice. These low critical temperatures, compared to those found in less frustrated systems such as the artificial square ice ${ }^{13}$, means that thus far only dynamic signatures of the phase transitions have been measured using indirect methods ${ }^{14}$. The real-space imaging of critical phenomena, however, has remained elusive, since this requires having nanomagnets large enough to be imaged and, although advances have been made to approach the critical temperatures using thermally superactive nanomagnets ${ }^{15}$, an alternative method is required to reach the critical phenomena.

One possibility to observe the critical phenomena is to tune the phase transition temperatures by modifying the interplay between geometrical frustration and the dipolar interaction. This can be achieved by altering the symmetry of the system, which at the same time changes the nature of the low-temperature phases. For example, analogous to using strain or chemical doping to modify the structure in microscopic systems ${ }^{16}$, in mesoscopic artificial spin systems one can compress the lattices 
or remove nanomagnets. For example, the Tetris or Shakti lattice ${ }^{17}$ can be obtained by removing nanomagnets from the artificial square ice. In these systems, the resulting unequal interactions give rise to rich phase diagrams and tunable phase transition temperatures ${ }^{9}$. Modifications to the square lattice have also been carried out by adding magnetic disks representing $X Y$ spins $^{18}$ and by creating elevated plateaus ${ }^{19,20}$, or to the artificial kagome spin ice by introducing notches in a connected system $^{21}$. Despite the predicted rich phase diagrams and the possibility of implementing thermallyactive nanomagnets to study them, there have not been any real-space temporal measurements of the phase transitions, only observations of relaxation towards thermal equilibrium ${ }^{22}$.

Here we focus on an archetypal highly frustrated system, the artificial kagome spin ice, which has two predicted phase transitions $s^{23,24}$. The kagome spin ice has a low energy six-fold degenerate vertex state, obeying either a two-in/one-out (vertex charge $q$ ) or one-in/two-out (vertex charge $-q$ ) ice rule, so that in a given temperature range it exhibits only ice-rule obeying moments (the so-called ice sector) with an extensive degeneracy. This degeneracy is lifted for dipolar interacting nanomagnets giving rise to two long-range ordered phases. As the temperature is reduced below the critical temperature $T_{\text {crit }}^{\text {charge }}=0.35 J_{\mathrm{NN}}$, there is a phase transition to a vertex-charge ordered state where $J_{\mathrm{NN}} \propto \mathrm{m}^{2} / \mathrm{a}^{3}$ is the nearest-neighbour (NN) interaction strength between nanomagnets of magnetic moment $\mathrm{m}$ separated by a centre-to-centre distance a (see Fig. 1a). As the temperature is further decreased to $\mathrm{T}_{\text {crit }}{ }^{\mathrm{RO}}=0.12 J_{\mathrm{NN}}$, the kagome spin system transitions to a long-range spin- and charge-ordered ground state (LRO). Within the ice-sector, the phase where $T>T_{\text {crit }}$ charge is referred to as Spin Ice 1 (SI-1) and can be viewed as a cooperative paramagnet or spin liquid ${ }^{25}$. The charge ordered regime of $T_{\text {crit }}{ }^{\mathrm{RO}}<\mathrm{T}$ $<\mathrm{T}_{\text {crit }}$ charge is referred to as the Spin Ice 2 (SI-2) phase and involves spin fragmentation and partial magnetic long range ordering ${ }^{26}$. For the lowest temperature regime of $T<\mathrm{T}_{\text {crit }^{\mathrm{L} O}}{ }^{\mathrm{RO}}$, a long-range ordered magnetic ground state is achieved. However, thus far, only the SI-1 phase has been directly observed in real-space $22,25,27,28$.

Rather than relying on weak and distant dipolar interactions to reach the low-temperature magnetic structure phases, we extend the tunability of this system by controlling the degeneracy strengths at the vertices. For this, we alter the six-fold vertex degeneracy through a structurally-induced modification with carefully designed magnetic bridges to give a broad range of degeneracy strengths (see Fig. 1b). This leads to a change in the global six-fold symmetry of the kagome lattice to that of a two-fold symmetry, thereby changing the universality class of the associated phase transitions. Because of this, we are able to probe the real space correlations of the bridged artificial kagome spin ice as a function of temperature, reaching the predicted long-range spin and charge-ordered magnetic ground state configuration of the artificial kagome spin ice. In this long-range ordered ground state, two out of three rings of nanomagnets have moments that form loops of head-to-tail moments, while the third ring has pairs of moments pointing head-to-head or tail-to-tail. By judiciously placing bridges at the vertices, the formation of moment loops, and therefore the long-range order, is facilitated.

We lithographically introduce three connecting magnetic bridges between nearest-neighbour nanomagnets at the vertex as shown in Fig. $1 \mathrm{a}$, and the fulfilment of the ice-rule requires the formation of a domain wall in one of the bridges. The energy of the domain wall is proportional to the width of the bridge in which it is formed, so the domain wall most likely appears in the thinnest bridge (see SM Fig. 1). This allows us to tune the strength of the degeneracy at each vertex by fabricating magnetic bridges of different widths. We restrict ourselves to two bridge widths, as shown in Fig. 1a, where one bridge has width $w_{a}$, which we vary between $0 \mathrm{~nm}$ and $100 \mathrm{~nm}$, while the other two bridges 
have a width $w_{\mathrm{b}}$ that is kept constant at $25 \mathrm{~nm}$. The artificial kagome spin ice is then recovered when $w_{\mathrm{a}}=w_{\mathrm{b}}=25 \mathrm{~nm}$, albeit with enhanced nearest neighbour interactions. Using MuMax ${ }^{29}$, we perform micromagnetic simulations to determine the interaction strengths $J_{\mathrm{i}}$ between two nanomagnets as a function of the bridge width. In Fig. $1 \mathrm{~b}$, we show the resulting interaction strengths $J_{\mathrm{a}}$ and $J_{\mathrm{b}}$ between nearest-neighbours connected via a bridge of width $w_{\mathrm{a}}$ or $w_{\mathrm{b}}$, respectively. The degeneracy strength is then defined as the ratio between the different nearest neighbour interaction strengths $J_{a} / J_{b}$ and is given in Fig. 1b.

In order to experimentally determine the effect of the bridge dimensions on the equilibrium magnetic states, we performed a thermal protocol on the bridged artificial kagome spin ice with different bridge dimensions by heating to $430 \mathrm{~K}$ and subsequently cooling to room temperature in half an hour. We used synchrotron $x$-ray photoemission electron microscopy (XPEEM) with $x$-ray magnetic circular dichroism (XMCD) to image the frozen-in magnetic configurations. In total, we have characterised 20 different bridged artificial kagome spin ices (see SM Fig. 2) with different bridge width ratios (i.e. different degeneracy strengths $J_{a} / J_{b}$ ) in order to determine the condition for which a phase transition from the SI-1 to the LRO phase can be observed. In Fig. 2, we show SEM images of four representative experimental systems. The coloured dots at the centre of the hexagonal rings of nanomagnets indicate loops of head-to-tail moments. Their colour indicates that the nanomagnets are connected by bridges of width $w_{\mathrm{a}}$ (green) or $w_{\mathrm{b}}$ (orange), or that $w_{\mathrm{a}}=w_{\mathrm{b}}$ (white) for equal interactions, and the size of the dots reflects the corresponding bridge width ratios $w_{\mathrm{a}} / w_{\mathrm{b}}$.

For the first time, long-range magnetic ordering of the kagome spin ice is observed in a modified thermally-active system with a two-fold degenerate vertex $\left(w_{\mathrm{a}}=0 \mathrm{~nm}\right.$, see SM Fig. 1$)$ with $J_{\mathrm{a}} / J_{\mathrm{b}}=$ 0.5187 , meaning $T_{\text {crit }}=640 \mathrm{~K}$ is much larger than $T_{B}=310 \mathrm{~K}$ (see green shaded region in Fig. 2a). For intermediate degeneracy strengths where $0.8<J_{a} / J_{b}<1$, the experimental system freezes in the middle of the continuous phase transition towards long-range magnetic order $\left(T_{B} \approx T_{\text {crit }}\right.$ ), as shown in Fig. 2b. In Fig. 2c, we show the degenerate artificial kagome spin ice with $J_{a} / J_{b}=1$ that freezes in the known SI-1 phase. In Fig. $2 d$, for the system with $J_{a} / J_{b}>1$ consisting of four-fold degenerate vertices $\left(w_{\mathrm{a}}>25 \mathrm{~nm}\right.$, see SM Fig. 1), a triangular ordering emerges of flux closure loops embedded in an icerule obeying background. This system exhibits a cross-over to the triangular arrangements of flux closure loops, but displays no phase transition as $T_{B}>T_{\text {crit. }}$. These results illustrate the effect asymmetric vertex interactions can have on the global magnetic ordering of the bridged artificial kagome spin ice, with the broader bridges resulting in loops of head-to-tail moments. The system with one thick bridge and two thinner bridges shown in Figure $2 \mathrm{~b}$ leads to local ordering but the long-range dipolar interactions are not yet strong enough to ensure a system spanning long-range magnetic ordering. The long-range ordered ground state of the kagome system is therefore only achieved in the field-of-view of the PEEM $\left(20 \times 20 \mu \mathrm{m}^{2}\right)$ when $w_{\mathrm{a}}<12 \mathrm{~nm}$ and $J_{\mathrm{a}} / \mathrm{J}_{\mathrm{b}}<0.7484$.

In order to visualise the extent of the long-range order, we compute the magnetic structure factor (MSF, see methods for details) from experimental spin configurations for various degeneracy strengths and these are shown in Fig. 2e. For $J_{a} / J_{b}=0.5187$, the fully ordered system gives rise to six distinct peaks in the MSF of equal intensity. As $J_{a} / J_{b}$ increases, this ordering remains until $J_{a} / J_{b}=0.86$ -0.88 , where a splitting of the peaks is observed as the system transitions from the LRO phase to the SI-1 phase. The peak splitting is a result of domain formation with a characteristic length scale of 12 $\mu \mathrm{m}$, which we have also observed in Monte Carlo simulations. This allows us to conclude that the peak 
splitting corresponds to reaching equilibrium magnetic states at the critical temperature of the phase transition ( $\left.T_{B}=T_{\text {crit }}\right)$.

We now observe how a system with $J_{a} / J_{b}=0.5187$ evolves to the long-range ordered magnetic state shown in Fig. $2 a$ by tracking the spatial correlations. This system has $w_{\mathrm{a}}=17 \mathrm{~nm}$ and $w_{\mathrm{b}}=25 \mathrm{~nm}$, and a thickness of $3.1 \mathrm{~nm}$ giving a blocking temperature above room temperature $T_{B}=310 \mathrm{~K}$. Starting from the as-grown configuration, we follow both the moment and charge configurations of such a system over several hours upon in-situ heating (see Methods). In Fig. 3a, we give the XMCD contrast images (see SM Video 1 for the full series), obtained as a function of time at constant temperature $\mathrm{T}=320 \mathrm{~K}$, tracking the charge ordering (yellow dots) of the bridged artificial kagome system. The system starts from an initial frozen as-grown out-of-equilibrium ice-rule obeying phase with mainly disordered charges (indicated by the blue dots), which then relaxes towards its equilibrium long-range ordered phase. In Fig. 3b, the corresponding formation of moment loops is given with red and magenta dots indicating left and right-handed chirality of the loops.

From these experimental PEEM images, we extract the time-dependent ordering of the spins and charges using a correlator analysis. Comparing, for instance the $2^{\text {nd }}$ neighbour spin order correlator $C_{\alpha \nu}$ in Fig. $3 c$ to the nearest-neighbour charge ordering in $C_{A B}$ in Fig. $3 d$, we see that spin order is achieved earlier than charge order as indicated by the black dotted lines. A stronger effect is seen in $C_{\alpha \delta}$, which reaches its equilibrium value $(-1 / 3)$ within 2 hours, indicating the fast ordering of spins that are not necessarily nearest-neighbours. This spin ordering is associated with the formation of headto-tail loops of moments and is in contrast to the ordering dynamics observed in the degenerate artificial kagome lattice ${ }^{22}$ where charge ordering occurs before spin ordering. This can be attributed to the asymmetric placement of interaction modifiers favouring the formation of loops of moments, while an average stronger nearest-neighbour interaction only partially increases the rate of charge ordering. Hence, the driving force for spin order (the nanoscale bridges) modifies the energy landscape and the ordering mechanism.

The quantitative temperature dependence of spin and charge correlators of our bridged kagome artificial spin ice is determined using Monte Carlo (MC) simulations with an appropriately modified nearest-neighbour interaction energy. From this, we estimate the spin and charge correlators as a function of temperature for various degeneracy strengths $J_{\mathrm{a}} / \mathrm{J}_{\mathrm{b}}$. Next, we fit the experimental spin and charge correlator dependence (SM Fig. 3ab) to the temperature dependence found using MC simulations (SM Fig. 3cd). Utilising a correlator analysis ${ }^{26,30}$, we determine the equilibrium temperature $\mathrm{T}_{\text {fit }}$ to be $310 \mathrm{~K}$, which coincides with the experimentally determined blocking temperature $T_{B}=310 \mathrm{~K}$. No further fitting of the $M C$ simulations was required to establish the same correlator trends with $J_{\mathrm{a}} / J_{\mathrm{b}}$. This allows us to directly convert the reduced temperatures from $\mathrm{MC}$ simulations to experimental temperature values.

Interestingly, a sharp change is observed in the spin and charge correlators at $J_{a} / J_{b}=0.87$ (see SM Fig. 3 ), indicating the onset of a continuous phase transition from the LRO phase to the SI-1 phase, which correlates with the observed peak splitting in the MSFs seen in Fig. 2e. The full set of XMCD contrast images as a function of $J_{a} / J_{b}$ (see SM Fig. 2) allows us to directly observe the phase transition. The phase transition can also be imaged using temperature-dependent XPEEM experiments, which allow the direct observation of temperature-dependent spin fluctuations at $T_{\text {crit. }}$ In order to achieve this, we use an artificial kagome spin ice with bridges such that $T_{B}$ is close to but just below $T_{\text {crit, }}$ which is at 
$J_{\mathrm{a}} / J_{\mathrm{b}}=0.847\left(w_{\mathrm{a}}=18 \mathrm{~nm}\right.$ and $\left.w_{\mathrm{b}}=25 \mathrm{~nm}\right)$. At this degeneracy strength, the critical temperature is 340 $\mathrm{K}$, while the blocking temperature is $310 \mathrm{~K}$, which is therefore perfectly suited to using the limited temperature range available in the XPEEM. Starting at $T_{B}$, we then measure a series of images taken at various temperatures above $T_{B}$, and compute the experimental MSF at each temperature by averaging over 100 moment configurations extracted from the XMCD contrast images (see Fig. 4a). Here, we can see that the peak splitting occurs upon heating as was observed in the $J_{a} / J_{b}$ series. In order to determine the universality class of the phase transition, we perform MC simulations of a system with $J_{a} / J_{b}=0.85$ and confirm the critical exponents. We find that the phase transition does indeed belong to the $2 \mathrm{D}$ Ising universality class (see Fig. 4b) with $v=1.0, y=7 / 4, \alpha=0, \beta=1 / 8$ (see SM Fig. 4 for detailed scaling graphs for various $J_{a} / J_{b}$ systems). The simulated temperature-dependent MSF in Fig. 4c confirms the peak splitting at $T_{\text {crit }}$ as a clear sign that it is possible to measure the phase transition in real space and time. The spin configurations in time at various temperatures are given in SM Videos 2 to 4. In these videos, it can be seen that domains of the two degenerate ground states are formed as the temperature is increased. It is the reduction in domain sizes that causes the observed peak splitting in the MSFs, from which the characteristic length scale is approximately 11 $\mu \mathrm{m}$.

We can now use the MC simulations to determine the complete phase diagram of the bridged artificial kagome spin ice as a function of the degeneracy strength $J_{\mathrm{a}} / J_{\mathrm{b}}$ and temperature (Fig. 5). In order to determine the critical temperatures, we use a Binder $4^{\text {th }}$ order cumulant analysis (see SM Fig. 4). The phase transition temperature $T_{\text {crit }}$ is found to be inversely proportional to $J_{a} / J_{b}$ (black crosses in Fig. 5) and can be controlled to be above, below, or at the superparamagnetic blocking temperature (blue horizontal line in Fig. 5). As expected, at $J_{a} / J_{b}=1$ the system exhibits the two phase transitions of the degenerate artificial kagome spin ice ${ }^{23,24}$ (from the SI-1 to SI-2 phase, and from SI-2 to the LRO phase). For decreasing values of $J_{\mathrm{a}} / J_{\mathrm{b}}, T_{\text {crit }}$ increases from the SI-1 to SI-2 critical temperature at $J_{\mathrm{a}} / J_{\mathrm{b}}=1$, now reflecting the $2 \mathrm{D}$ Ising transition from the SI-1 to the LRO phase. Underlying this transition are shortrange fluctuations in both spin order and charge order as probed by the correlators shown in Fig. $3 \mathrm{c}$ and $3 \mathrm{~d}$. These indicate that an increase in local charge order precedes that of local spin order for $0.8474<J_{a} / J_{b}<1$, whereas for $0.5187<J_{a} / J_{b}<0.8474$ the reverse is seen as given by the red and black points in Fig. 5 at which $\mathrm{C}_{\mathrm{AB}}=-0.8$ and $\mathrm{C}_{\alpha \gamma}=-0.8$. These specific spin and charge correlators are the equilibrium values at the critical temperatures of the two phase transitions occurring in the degenerate artificial kagome spin ice. For the latter regime, observed experimentally in Fig. 3 for $J_{a} / J_{b}$ $=0.5187$, spin order precedes charge order due to the initial formation of unordered loops of headto-tail moments which subsequently order. Interestingly, the temperature dependence of $C_{\alpha \gamma}=-0.8$ smoothly asymptotes to the temperature associated with the transition from the SI- 2 to the LRO phase of the degenerate kagome system as $J_{a} / J_{b}$ approaches unity, whereas the $C_{A B}=-0.8$ line limits to its Spin Ice 1 to Spin Ice 2 critical temperature indicating that, whilst the Monte Carlo simulations reveal one phase transition to long-range order for $J_{\mathrm{a}} / J_{\mathrm{b}}<1$, there exist strong fluctuations representative of the SI-2 to LRO transition when close to $J_{a} / J_{b}=1$ (see shoulder present at $\mathrm{Ja} / \mathrm{Jb}=$ 0.99 in the susceptibility in SM Fig. 4).

In conclusion, nearest-neighbour interactions are modified when nanoscale bridges are introduced to break the symmetry and to continuously tune degeneracy strengths in an otherwise highly degenerate artificial kagome spin ice system. By varying the widths of the bridges, we can control the critical temperature and, by increasing the critical temperature above the blocking temperature, we are able to observe the long-range magnetic ground state of the artificial kagome spin ice in a system with 
modified symmetry. This is achieved because, by the lifting of the vertex degeneracy with patterned magnetic bridges, we change the nearest-neighbour interactions asymmetrically, so changing the global symmetry from a three-state Potts system ${ }^{24}$ to that of $2 \mathrm{D}$ Ising. The ordering is tracked by determining the spin and charge correlators over time and we observe a reversal of the driving forces for spin and charge ordering compared to the degenerate artificial kagome spin ice. Furthermore, on determining the spin and charge correlators as a function of degeneracy strength, we observe a structurally induced continuous phase transition whose critical temperature depends inversely on the degeneracy strength. The phase transition observed in bridged artificial kagome spin ice is confirmed from temperature-dependent data, both experimentally and from Monte Carlo simulations in combination with micromagnetic simulations. Here, a peak splitting in the magnetic structure factors serves as the indicator for $T_{B}=T_{\text {crit }}$ and confirms the measurement of the critical phenomena associated with the phase transition.

We have demonstrated that artificial spin systems can be tuned with nanoscale magnetic bridges, allowing for the control of the degeneracy of the long range ordered ground state through a change in global symmetry, and through a modification of the level of frustration of the spin configurations obeying the ice rule. Such fine control of the magnetic energy landscape allows for the creation of artificial spin systems whose equilibrium thermal properties can be measured experimentally. Whilst the focus of the present work has been on the equilibrium properties of an ordered system, nanoscale magnetic bridges of varying sizes would allow for a controllable introduction of disorder, raising the degeneracy further and introducing a more complex energy landscape whose thermal properties could exhibit glass-like properties ${ }^{31,32}$ that can be probed in real-space.

\section{Methods}

Sample fabrication. Our sample consists of a set of arrays of nanomagnets made of Permalloy with different thickness, and with various bridge width dimensions on a silicon substrate. The sample was prepared by spin-coating a Si(001) 10 by $10 \mathrm{~mm}^{2}$ substrate with Poly(methyl methacrylate) (PMMA) resist. The designed patterns are then exposed using the Vistec EBPG 5000PlusES electron beam writer at $100 \mathrm{keV}$ accelerating voltage. The exposed areas are developed using a mixture of MIBK and isopropanol in the ratio 1:3, rinsed with IPA, and spin-dried. A wedge (variable thickness sample) of Permalloy $\mathrm{Ni}_{80} \mathrm{Fe}_{20}$ is deposited using thermal evaporation (base pressure $1 \times 10^{-6} \mathrm{mbar}$ ) and is capped with a $2.5 \mathrm{~nm}$-thick layer of aluminium to prevent oxidation. Ultrasound-assisted lift-off in acetone is used to remove the undeveloped resist leaving only the nanomagnets and bridges on the substrate. The resulting nanomagnets have lengths $L=450 \mathrm{~nm}$ and widths $W=150 \mathrm{~nm}$, and a variable thickness from $0 \mathrm{~nm}$ to $7 \mathrm{~nm}$ Permalloy over a distance of $7 \mathrm{~mm}$ on the substrate.

$X$-ray photoemission electron microscopy (XPEEM). Magnetic imaging was performed at the Surface/Interface: Microscopy (SIM) beamline of the Swiss Light Source, Paul Scherrer Institute, Villigen PSI, Switzerland ${ }^{33}$. Using the PEEM endstation ${ }^{34}$, the magnetic contrast is obtained by exploiting $x$-ray magnetic circular dichroism $(X M C D)$ and dividing the images obtained with right- and left-circularly polarised $x$-rays at the iron $\mathrm{L}_{3}$ edge ${ }^{35}$. The resulting XMCD contrast is proportional to the projection of the magnetic moments onto the $x$-ray propagation vector, with moments pointing towards (away from) the incoming $x$-rays appearing bright (dark) with a sensitivity and resolution that allows for the determination of the entire magnetic configuration.

Micromagnetic simulations. Micromagnetic simulations are performed using bulk material parameters for Py nanomagnets with a magnetisation at saturation $M_{\text {sat }}=790 \mathrm{kA} / \mathrm{m}$, an exchange stiffness constant $A_{e x}=13 \mathrm{pJ} / \mathrm{m}$, and zero magnetocrystalline anisotropy. The cell sizes are $1 \times 1 \times 3 \mathrm{~nm}^{3}$. The energy equation for the two interacting nanomagnets can subsequently be solved to find all interaction strengths $\left(J_{\mathrm{ij}}\right.$ 's) as a function of the bridge width using a method similar to those used our 
other works ${ }^{15,36}$. The vertex energies are obtained from different configurations by relaxing the magnetic configuration of three nanomagnets to give a magnetic state according to the various types. Monte Carlo simulations. We performed Monte Carlo (MC) simulations to determine the behaviour of arrays of nanomagnets in a bridged artificial kagome spin ice on a larger scale by modelling them as individual point dipoles with Ising degrees of freedom that point along the long axis of the nanomagnets. Here we compute the temperature-dependent equilibrium configurations for various system sizes to determine critical exponents, and perform a Binder $4^{\text {th }}$ order cumulant analysis to determine critical temperatures, as in prior works ${ }^{13}$. The order parameter used in the Binder $4^{\text {th }}$ order cumulant analysis is the AFM diffraction peak $Q_{A F M}=\left(\frac{2}{\sqrt{3}} \frac{\pi}{2 a}, \frac{2}{3} \frac{\pi}{2 a}\right)$, specifically $M^{2}=$ $\frac{1}{2} \sum_{Q_{A F M}}\left|\mathbf{M}_{\mathbf{Q}}\right|^{2}$ and the Binder $4^{\text {th }}$ order cumulant is $1-\left\langle\sqrt{M^{4}}\right\rangle /\left\langle\sqrt{M^{2}}\right\rangle^{2} / 3$. We start with a dipolar Hamiltonian that is extended to the bridged artificial kagome spin ice by including the tabulated nearest-neighbour interaction strengths determined using micromagnetic simulations.

Magnetic structure factors. In order to obtain information about the long-range ordering and the phase transition, we calculate the magnetic structure factors $\mathbf{M}_{Q}=\frac{1}{\sqrt{\mathrm{N}}} \sum_{\mathrm{N}} \widehat{\mathbf{m}} \exp \left(i \mathbf{r}_{\mathbf{j}} \cdot \mathbf{Q}\right)$, which is defined as the Fourier transform of the magnetic spin configuration. Here $\mathbf{Q}$ can be the discrete values $\left(\frac{2 \pi}{n a} n_{x}, \frac{2 \pi}{n a} n_{y}\right)$ with $n_{i}$ ranging from $-n / 2$ to $n / 2$.

\section{Acknowledgements}

K.H., S.H.S., H.A., and L.J.H. acknowledge support from the Swiss National Science Foundation (Projects No. 200020_172774). Part of this project was performed at the Surface/Interface: Microscopy (SIM) beamline of the Swiss Light Source, Paul Scherrer Institute, Villigen PSI, Switzerland.

\section{Contributions}

K.H. and L.J.H. conceived the project. K.H. fabricated the samples, and designed and performed the experiments with support from H.A., Z.L., S.P., and A.K. K.H. analysed and interpreted the data with support from P.M.D. and L.J.H., and P.M.D. wrote the Monte Carlo code used by K.H. to perform the correlator analysis and phase diagram determination with support from S.H.S. K.H. wrote the manuscript with input from all authors.

\section{Competing Interests}

The authors declare no competing financial interests.

\section{Data availability}

The data that support the findings in this study are available via the Zenodo repository 'to be added'.

\section{References:}

1. Heyderman, L. J. \& Stamps, R. L. Artificial ferroic systems: novel functionality from structure, interactions and dynamics. J. Phys. Condens. Matter 25, 363201 (2013).

2. Nisoli, C., Moessner, R. \& Schiffer, P. Colloquium: Artificial spin ice: Designing and imaging magnetic frustration. Rev. Mod. Phys. 85, 1473-1490 (2013).

3. Skjærv $\varnothing$, S. H., Marrows, C. H., Stamps, R. L. \& Heyderman, L. J. Advances in artificial spin ice. Nature Reviews Physics vol. 2 13-28 (2020).

4. Schiffer, P. \& Nisoli, C. Artificial spin ice: Paths forward. Appl. Phys. Lett. 118, (2021).

5. Perrin, Y., Canals, B. \& Rougemaille, N. Extensive degeneracy, Coulomb phase and magnetic monopoles in artificial square ice. Nature 540, 410-413 (2016).

6. Farhan, A. et al. Emergent magnetic monopole dynamics in macroscopically degenerate artificial spin ice. Sci. Adv. 5, eaav6380 (2019). 
7. Gilbert, I. et al. Emergent reduced dimensionality by vertex frustration in artificial spin ice. Nat. Phys. 12, 162-165 (2016).

8. Lao, Y. et al. Classical topological order in the kinetics of artificial spin ice. Nat. Phys. 14, 1-5 (2018).

9. Sklenar, J. et al. Field-induced phase coexistence in an artificial spin ice. Nat. Phys. 15, 191195 (2019).

10. Jensen, J. H., Folven, E. \& Tufte, G. Computation in artificial spin ice. in ALIFE 2018 - 2018 Conference on Artificial Life: Beyond Al 15-22 (2020). doi:10.1162/isal_a_00011.

11. Nomura, H. et al. Reservoir computing with dipole-coupled nanomagnets. Jpn. J. Appl. Phys. 58, (2019).

12. Hon, K. et al. Numerical simulation of artificial spin ice for reservoir computing. Appl. Phys. Express 14, (2021).

13. Sendetskyi, O. et al. Continuous magnetic phase transition in artificial square ice. Phys. Rev. $B$ 99, 1-11 (2019).

14. Anghinolfi, L. et al. Thermodynamic phase transitions in a frustrated magnetic metamaterial. Nat. Commun. 6, 8278 (2015).

15. Hofhuis, K. et al. Thermally superactive artificial kagome spin ice structures obtained with the interfacial Dzyaloshinskii-Moriya interaction. Phys. Rev. B 102, 180405 (2020).

16. Torrance, J. B., Lacorre, P., Nazzal, A. I., Ansaldo, E. J. \& Niedermayer, C. Systematic study of insulator-metal transitions in perovskites $\mathrm{RNiO3}(\mathrm{R}=\mathrm{Pr}, \mathrm{Nd}, \mathrm{Sm}, \mathrm{Eu})$ due to closing of chargetransfer gap. Phys. Rev. B 45, 8209-8212 (1992).

17. Shi, Y., Nisoli, C. \& Chern, G. W. Ice, glass, and solid phases in artificial spin systems with quenched disorder. Appl. Phys. Lett. 118, (2021).

18. Östman, E. et al. Interaction modifiers in artificial spin ices. Nat. Phys. 14, 375-379 (2018).

19. Perrin, Y., Canals, B. \& Rougemaille, N. Extensive degeneracy, Coulomb phase and magnetic monopoles in artificial square ice. Nature 540, 410-413 (2016).

20. Farhan, A. et al. Emergent magnetic monopole dynamics in macroscopically degenerate artificial spin ice. Sci. Adv. 5, eaav6380 (2019).

21. Schánilec, V. et al. Bypassing Dynamical Freezing in Artificial Kagome Ice. Phys. Rev. Lett. 125, 057203 (2020).

22. Farhan, A., Derlet, P. M., Anghinolfi, L., Kleibert, A. \& Heyderman, L. J. Magnetic charge and moment dynamics in artificial kagome spin ice. Phys. Rev. B 96, 1-9 (2017).

23. Möller, G. \& Moessner, R. Magnetic multipole analysis of kagome and artificial spin-ice dipolar arrays. Phys. Rev. B - Condens. Matter Mater. Phys. 80, 2-4 (2009).

24. Chern, G. W., Mellado, P. \& Tchernyshyov, O. Two-stage ordering of spins in dipolar spin ice on the kagome lattice. Phys. Rev. Lett. 106, 1-4 (2011).

25. Rougemaille, N. \& Canals, B. Cooperative magnetic phenomena in artificial spin systems: spin liquids, Coulomb phase and fragmentation of magnetism--a colloquium. Eur. Phys. J. B 92, 62 
(2019).

26. Canals, B. et al. Fragmentation of magnetism in artificial kagome dipolar spin ice. Nat. Commun. 7, 1-6 (2016).

27. Qi, Y., Brintlinger, T. \& Cumings, J. Direct observation of the ice rule in an artificial kagome spin ice. Phys. Rev. B - Condens. Matter Mater. Phys. 77, 1-4 (2008).

28. Zhang, S. et al. Crystallites of magnetic charges in artificial spin ice. Nature 500, 553-557 (2013).

29. Leliaert, J. et al. Fast micromagnetic simulations on GPU - Recent advances made with mumax3. J. Phys. D. Appl. Phys. 51, 123002 (2018).

30. Chioar, I. A. et al. Kinetic pathways to the magnetic charge crystal in artificial dipolar spin ice. Phys. Rev. B - Condens. Matter Mater. Phys. 90, 1-5 (2014).

31. Saccone, M. et al. Towards artificial Ising spin glasses: Thermal ordering in randomized arrays of Ising-type nanomagnets. Phys. Rev. B 99, 224403 (2019).

32. Saccone, M. et al. Elevated effective dimension in tree-like nanomagnetic Cayley structures. Nanoscale (2019) doi:10.1039/c9nr07510k.

33. Flechsig, U., Patthey, L. \& Schmidt, T. Performance measurements at the SLS spectroscopy beamline. AIP Conf. Proc. 705, 316-319 (2004).

34. Le Guyader, L. et al. Studying nanomagnets and magnetic heterostructures with X-ray PEEM at the Swiss Light Source. J. Electron Spectros. Relat. Phenomena 185, 371-380 (2012).

35. Stöhr, J. et al. Element-Specific Magnetic Microscopy with Circularly Polarized X-rays. Science (80-. ). 259, 658-661 (1993).

36. Farhan, A. et al. Geometrical Frustration and Planar Triangular Antiferromagnetism in QuasiThree-Dimensional Artificial Spin Architecture. Phys. Rev. Lett. 125, 267203 (2020). 

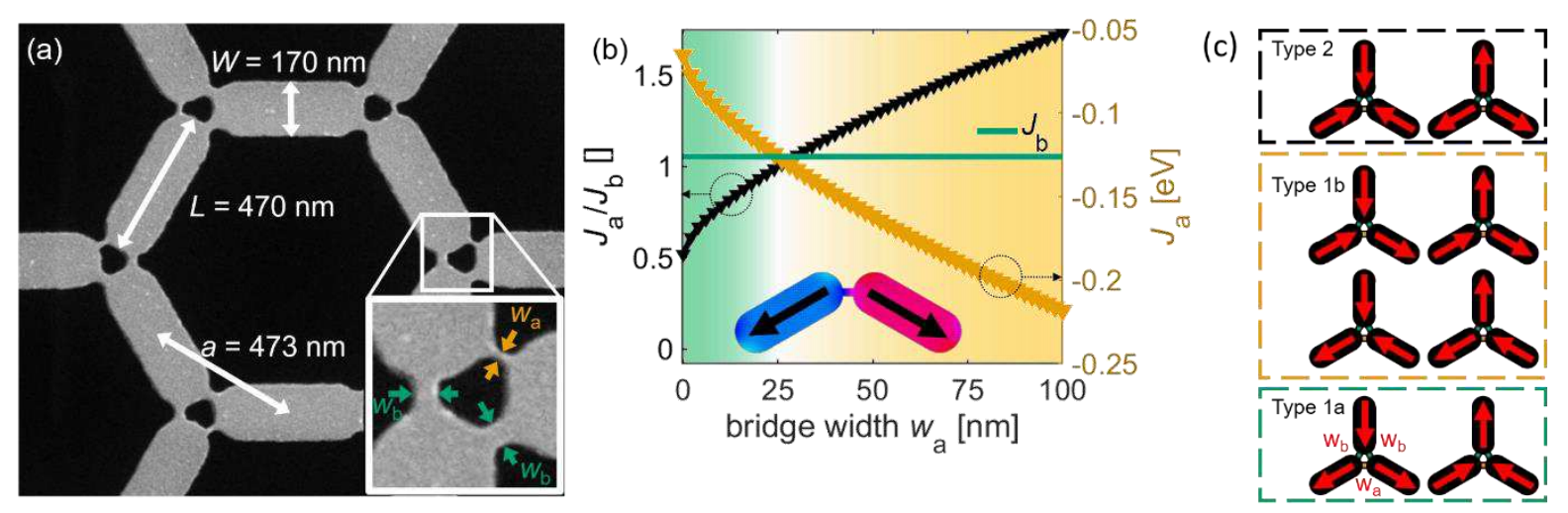

Fig. 1 | Sample geometry with corresponding pair-wise interaction strengths and vertex types.

a, Scanning electron microscope (SEM) image of part of an artificial kagome spin ice with bridges connecting the patterned $\mathrm{Ni}_{80} \mathrm{Fe}_{20}$ nanomagnets of dimensions length $L$, width $W$, and thickness $h$ of $470 \times 170 \times 3 \mathrm{~nm}^{3}$. The lattice parameter $a$ is $473 \mathrm{~nm}$, and a close-up of the asymmetric bridges at the vertex is shown in the inset. The bridge width $w_{\mathrm{a}}$ is varied from $0 \mathrm{~nm}$ to $100 \mathrm{~nm}$, while the bridge width $w_{b}$ is kept constant at $25 \mathrm{~nm}$. b. From micromagnetic simulations, the strengths of the interactions $J_{\mathrm{a}}$ and $J_{\mathrm{b}}$ between nearest-neighbour nanomagnets connected via a bridge of width $w_{\mathrm{a}}$ or $w_{b}$, respectively, are determined (right $y$-axis). The ratio between the different nearest-neighbour interaction strengths is defined as the degeneracy strength $\left(J_{a} / J_{b}\right.$ left $y$-axis). c, At $J_{a} / J_{b}=1$, the system represents the degenerate artificial kagome spin ice with a six-fold vertex degeneracy (Type $1 \mathrm{a}$ and $1 \mathrm{~b}$ vertex configurations are equal in energy). For $J_{\mathrm{a}} / J_{\mathrm{b}}<1$, green dashed box (green region in b), the vertex degeneracy is two-fold indicated as Type $1 \mathrm{a}$, while for $J_{\mathrm{a}} / J_{\mathrm{b}}>1$, orange dashed box (orange region in $\mathbf{b}$ ) results in a four-fold degenerate Type $1 \mathrm{~b}$ vertex. See SM Fig. 1 for the vertex energy simulations. 

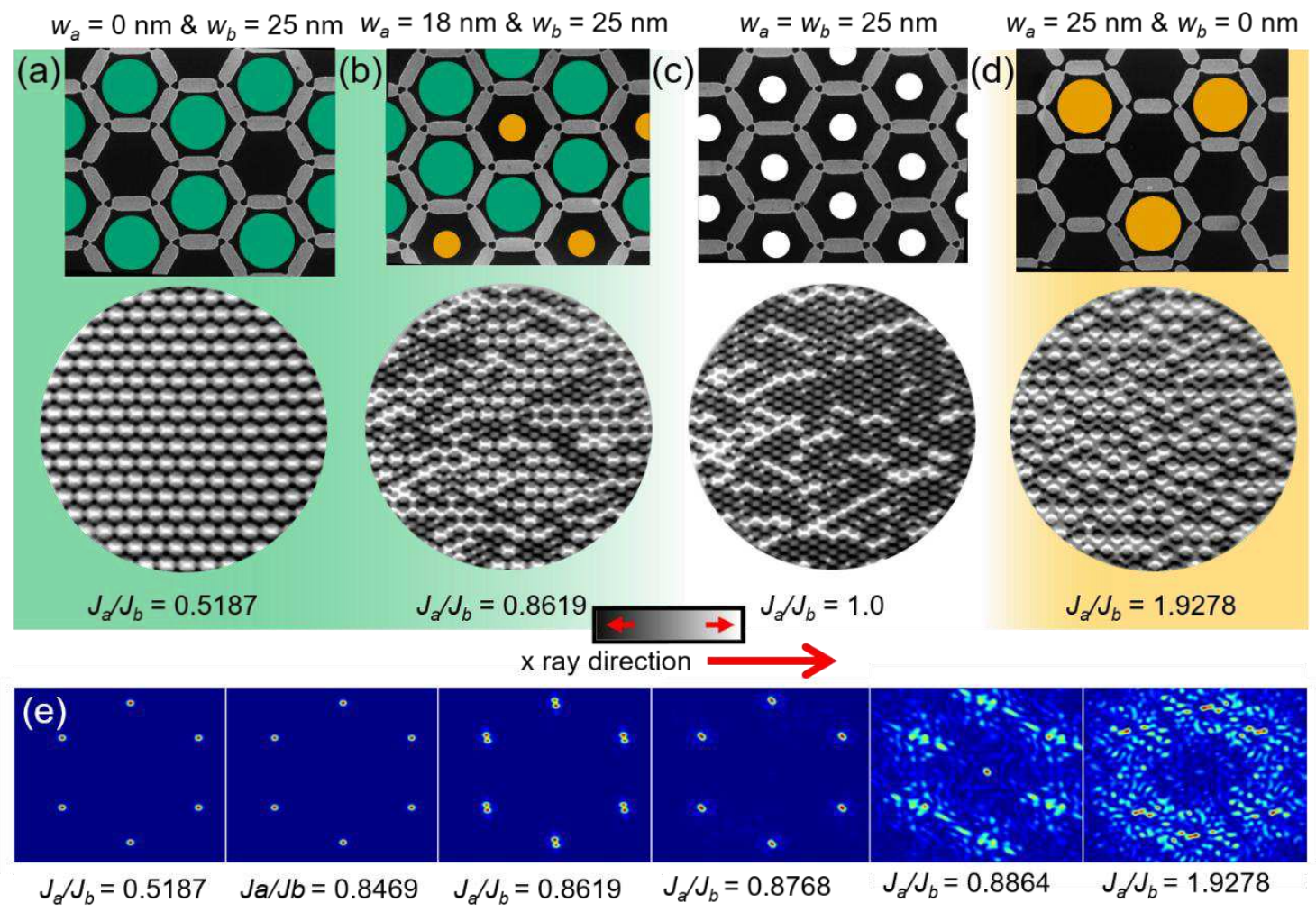

Fig. 2 | Magnetic configurations as a function of degeneracy strength.

SEM images of four selected experimental systems with the coloured dots indicating hexagonal rings of nanomagnets connected by bridges of width $w_{\mathrm{a}}$ (green) or $w_{\mathrm{b}}$ (orange) with the size of the dots indicating their respective widths. White dots indicate the degenerate artificial kagome spin ice with equal bridge widths $\left(w_{\mathrm{a}}=w_{\mathrm{b}}\right)$. The dots also correspond to the rings where loops of head-to-tail moments minimise the energy in an extended lattice. The samples are heated to $430 \mathrm{~K}$ and subsequently cooled to room temperature so that the moment configurations are frozen and imaged using XPEEM. $a$, The bridged artificial kagome lattice with $J_{\mathrm{a}} / J_{\mathrm{b}}=0.5187$ (green region) results in long-range magnetic ordering, while for $\mathbf{b}$, with intermediate degeneracy strengths $J_{a} / J_{b}=$ 0.8619 , the system freezes at the blocking temperature $\left(T_{B}=310 \mathrm{~K}\right)$, which is near the temperature of the phase transition from the SI-1 phase to long-range magnetic order. In $\mathbf{c}$, the artificial kagome spin ice with $J_{a} / J_{b}=1$ freezes in the SI-1 phase. This degenerate system is susceptible to extrinsic influences such as bias fields present in the experiment resulting in ordering of several moments towards the same direction seen as a dark contrast. At the same time, in $\mathbf{d}$, the system with $J_{\mathrm{a}} / J_{\mathrm{b}}>1$ results in a triangular ordering of loops of head-to-tail moments embedded in an ice-rule obeying background. e, Magnetic Structure factors for various $J_{a} / J_{b}$ showing a perfect long-range order in the left two panels $\left(J_{a} / J_{b}<0.847\right.$ with $\left.T_{B}<T_{\text {crit }}\right)$, then a peak splitting at the phase transition $\left(J_{a} / J_{b}=0.86-\right.$ 0.88 , where $T_{B}=T_{\text {crit }}$ and for a characteristic length scale of $12 \mu \mathrm{m}$ ). Finally, a diffuse partial ordering in the Spin Ice 1 phase emerges for $J_{a} / J_{b}=0.8864\left(T_{B}>T_{\text {crit }}\right)$ and is also seen for $J_{a} / J_{b}=1.9278$ that has a triangular ordering of moment loops but no long-range ordering as $T_{B}>T_{\text {crit. }}$ The XPEEM field-ofview of has a diameter of $20 \mu \mathrm{m}$. 

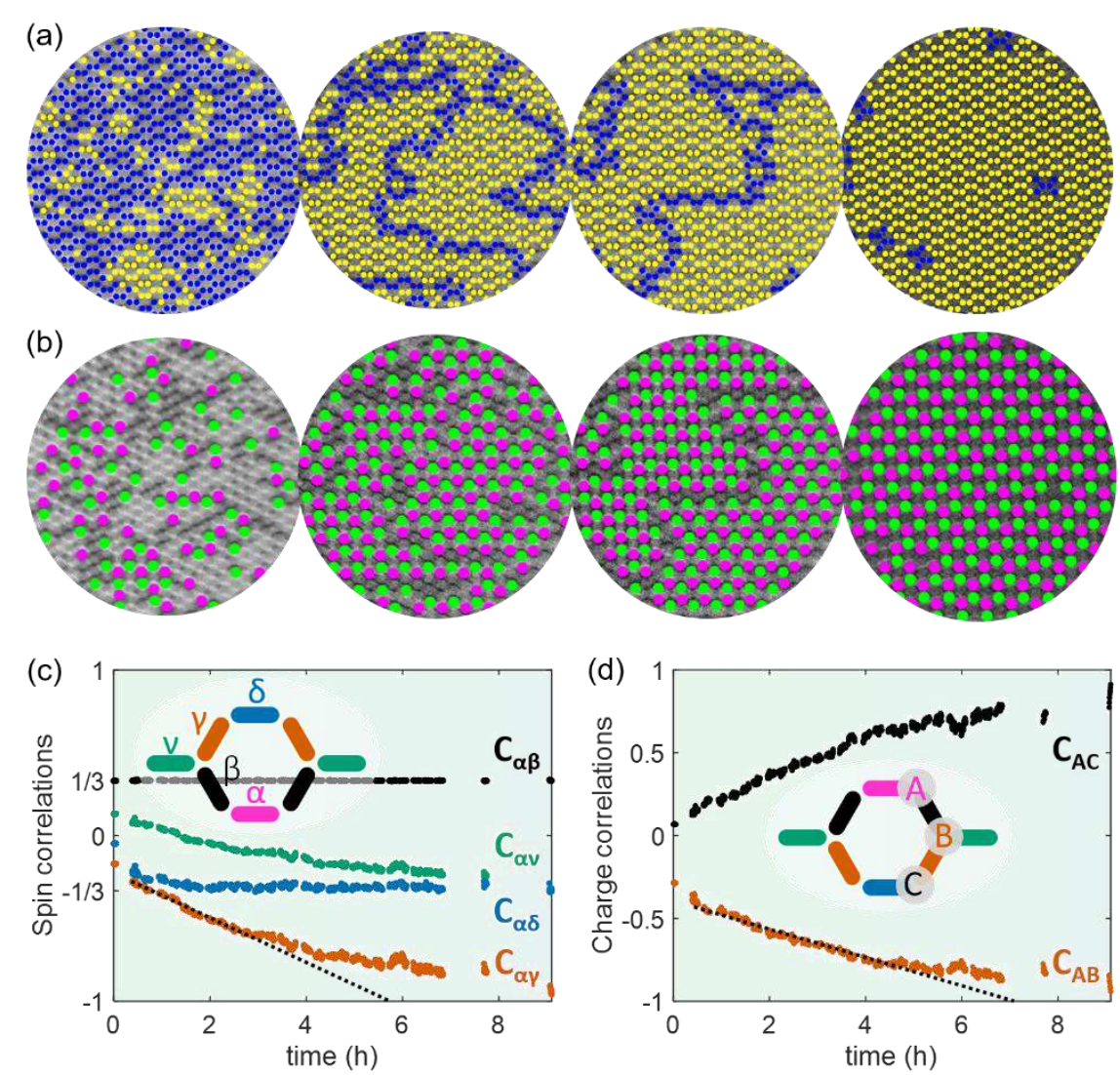

Fig. 3 | Relaxation to thermal equilibrium giving spin and charge order.

a, XMCD contrast images, obtained as a function of time at constant temperature $T=320 \mathrm{~K}\left(\mathrm{~T}_{\mathrm{B}}=310\right.$ $K)$, showing the thermal ordering of the bridged artificial kagome spin ice with $J_{a} / J_{b}=0.5187$, starting from an initial frozen as-grown out-of-equilibrium ice-rule obeying phase, which relaxes towards the equilibrium long-range ordered phase. The vertex charge ordering is indicated with yellow dots, while blue dots indicate disordered charges in a. The corresponding formation of moment loops is given in $\mathbf{b}$, indicated with magenta and green for left and right-handed chirality of the loops. The spin and charge correlators (see insets) are tracked over time in $\mathbf{c}$, and $\mathbf{d}$, extracted from a field-ofview of $20 \mu \mathrm{m}$. The equilibrium spin correlations of the long-range magnetic order of kagome spin ice are $C_{\alpha \beta}=1 / 3, C_{\alpha \nu}=C_{\alpha \delta}=-1 / 3$, and $C_{\alpha \gamma}=-1$, and the equilibrium charge correlations are $C_{A B}=-1$ and $C_{A C}=1$. See $S M$ Video 1 for the movie. 

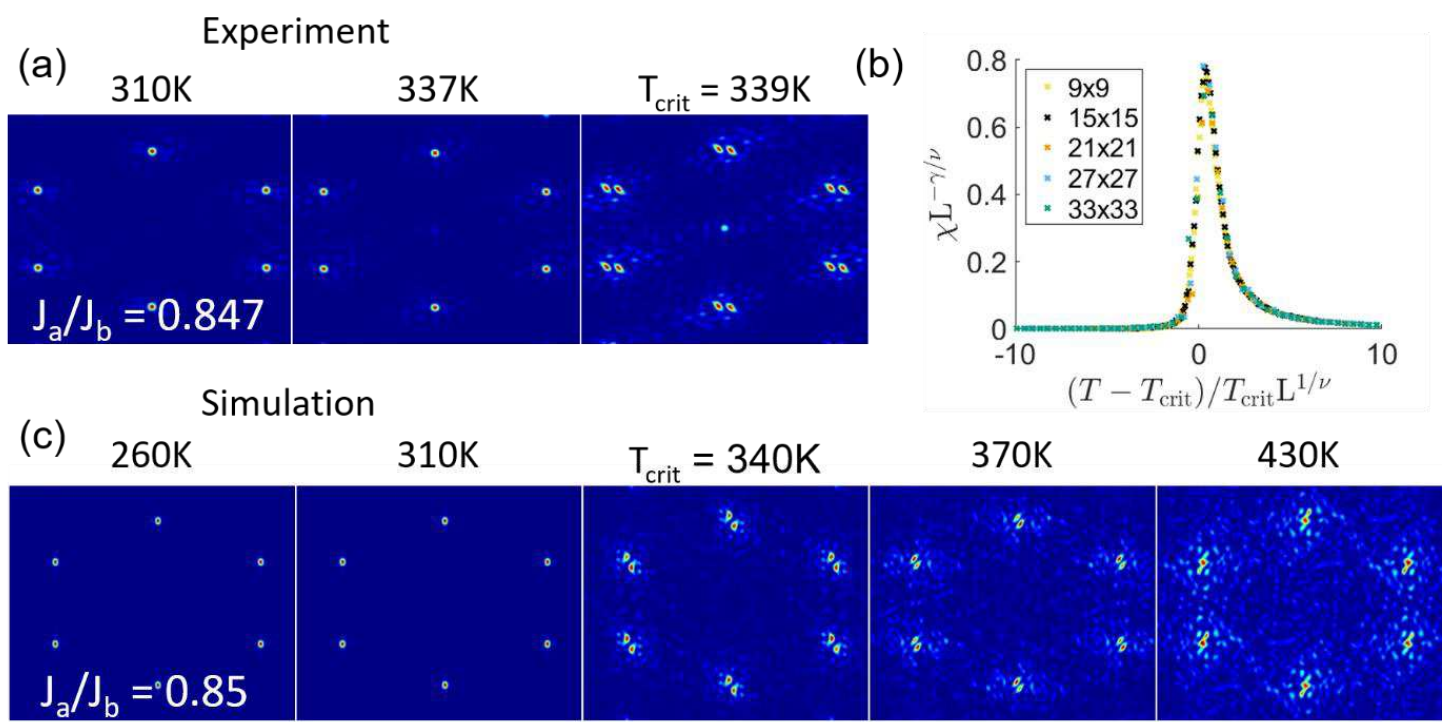

Fig. 4 | Peak splitting in magnetic structure factors upon heating to the phase transition.

a, Experimentally obtained temperature dependence of the magnetic structure factors (MSFs) extracted from the spin configurations obtained with XPEEM. The peak splitting occurs abruptly at the identified critical temperature of $339 \mathrm{~K}$. $\mathbf{b}$, Rescaled magnetic susceptibility obtained from Monte Carlo simulations using the $2 \mathrm{D}$ Ising universality critical exponents ( $v=1.0, y=7 / 4, \alpha=0, \beta=1 / 8$ ) indicating a good collapse of the simulated system size. System sizes are indicated as $L x L$ with $L=9$, $15,21,27$, and 33 corresponding to a system of $6 L^{2}$ spins. Here, $T_{\text {crit }}$ is obtained from a Binder $4^{\text {th }}$ order cumulant analysis (SM Fig. 5). c, MSFs of the spin configurations obtained from Monte Carlo simulations. In the simulations at $\mathrm{T}_{\text {crit }}=340 \mathrm{~K}$, a similar peak splitting is evident and is due to the formation of domains in the finite lattice (2646 of spins) with a characteristic length scale of $11 \mu \mathrm{m}$ within the XPEEM field-of-view of $20 \mu \mathrm{m}$. 


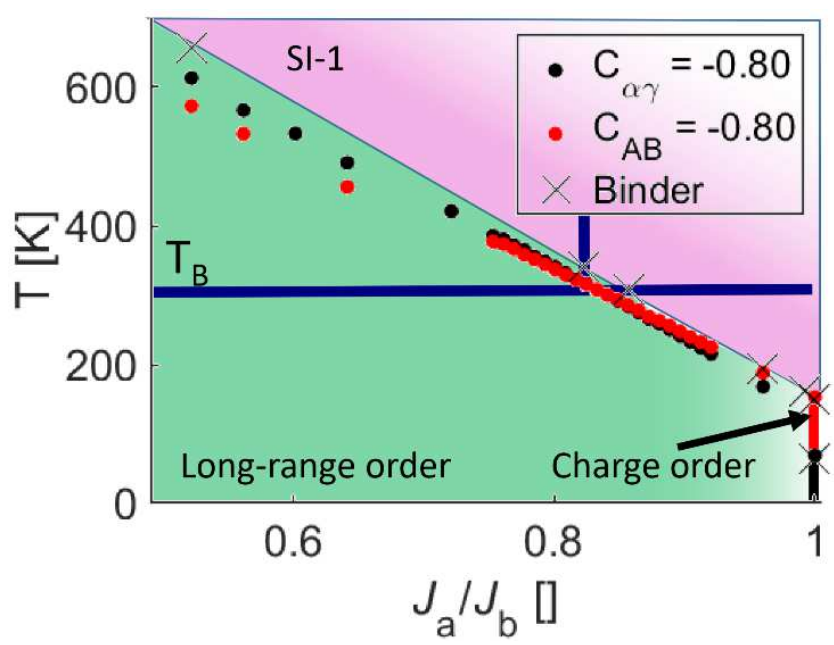

Fig. 5| Phase diagram of bridged artificial kagome spin ice.

Phase diagram obtained from Monte Carlo simulations, with a Binder $4^{\text {th }}$ order cumulant analysis employed to estimate $T_{\text {crit }}$ (black crosses) using the $J_{a} / J_{b}$ values obtained from micromagnetic simulations (see Fig. $1 \mathrm{~b})$. The spin correlator $\left(\mathrm{C}_{\alpha \gamma}=-0.80\right.$ - black dots) and charge correlator $\left(\mathrm{C}_{\mathrm{AB}}=-\right.$ 0.80 - red dots) are given as a function of degeneracy strength $J_{a} / J_{b}$, and indicate that the shortrange spin ordering precedes charge ordering for strong degeneracy breaking $\left(J_{a} / J_{b}<0.8\right)$. This is in contrast to the situation for $J_{a} / J_{b}>0.8$, including the degenerate artificial kagome spin ice at $J_{a} / J_{b}=1$, where charge order precedes spin order. The location of the experimental series of $J_{a} / J_{b}$ (from SM Fig. 3) with $T_{B}=310 \mathrm{~K}$ is given by the horizontal blue line, showing a crossing from the LRO phase (green region) to the SI-1 phase (magenta region) at $J_{a} / J_{b}=0.8619$, corresponding to a $T_{\text {crit }}$ of $310 \mathrm{~K}$. The location of the experimental series of temperature-dependent XPEEM data is given by the vertical blue line at $J_{a} / J_{b}=0.8474$ and crosses from the long-range ordered to the SI- 1 phase at the phase transition $\left(T_{\text {crit }}=340 \mathrm{~K}\right)$. For the system with $J_{a} / J_{b}=1$, there are two phase transitions from the $\mathrm{SI}-1$ to SI-2 phase (charge order), and from the SI-2 to the long-range ordered phase of the degenerate artificial kagome spin ice at $\mathrm{T}_{\text {crit }}=149 \mathrm{~K}$ and $56 \mathrm{~K}$, respectively. The other bridged artificial kagome spin ices at $J_{\mathrm{a}} / J_{\mathrm{b}}=[0.5,0.85,0.96,0.99]$ exhibit only one phase transition at $\mathrm{T}_{\text {crit }}=$ $[656.5,309,194,160.4,149] \mathrm{K}$, respectively. 


\section{Supplementary Files}

This is a list of supplementary files associated with this preprint. Click to download.

- HofhuisbridgedASISM.docx

- SMVideo1XMCDspintime5Hz.mp4

- SMVideo2XMCDseries310K.mp4

- SMVideo3XMCDseries337K.mp4

- SMVideo4XMCDseries339K.mp4 